\title{
Planning of Fibre to the Curb using G.Fast in multiple roll-out scenarios
}

\author{
Frank Phillipson \\ TNO, Delft, the Netherlands \\ Email: frank.phillipson@tno.nl
}

\begin{abstract}
In this paper we present a simple framework for planning options when deploying Fibre to the Curb, using G.Fast as technology. We present a framework of eight possible planning options, roll-out scenarios, coming from three main planning choices. We elaborate the mathematical approach of each of these eight options, using combinations of existing methods. We also show the results of a real life case, rolling out Fibre to the Curb in Amsterdam and The Hague, resulting in an example of the calculation time needed and the indication of the costs of such a roll-out.
\end{abstract}

Index Terms-G.Fast, Fibre to the Curb, access network planning, telecommunications

\section{INTRODUCTION}

In the near future new internet services will be so demanding in bit rate that they easily consume a bandwidth of hundreds of $\mathrm{Mb} / \mathrm{s}$, they probably include many high definition video channels simultaneously. To deliver these services to consumer's homes the use of fibre will be inevitable but this does not necessarily mean that fibre is to be deployed all the way to a point into the home, Full Fibre to the Home (Full $\mathrm{FttH}$ ). An alternative is bringing fibre up or near to the home, reusing existing copper cables. The copper technology that is required for such a Hybrid FttH solution with sufficient bandwidth is currently developed and is named G.Fast. First results of this development make it plausible that Hybrid FttH using G.Fast is technically feasible up to $1 \mathrm{~Gb} / \mathrm{s}$. For this work look at the website of the CELTIC/4GBB project [1].

We distinguish four topology types (see Fig. 1):

1) Full Copper: services are offered from the Central Office (CO) over a copper cable, using ADSL or VDSL techniques.

2) Fibre to the Cabinet (FttCab): the fibre connection is extended to the cabinet. From the cabinet the services are offered over the copper cable, using VDSL or G.Fast techniques.

3) Hybrid Fibre to the Home (Hybrid FttH): services are offered from a Hybrid FttH Node, which is connected by fibre, close to the customer premises, in the street or in the building.

4) Full Fibre to the Home (Full FttH): the fibre connection is brought up to the customer premises.

In this paper we look at the planning of the Hybrid FttH variant, where the fibre is brought to a place in the street, also known as Fibre to the Curb (FttCurb). To realize FttCurb using G.Fast a next step in bringing fibre to the houses is needed.

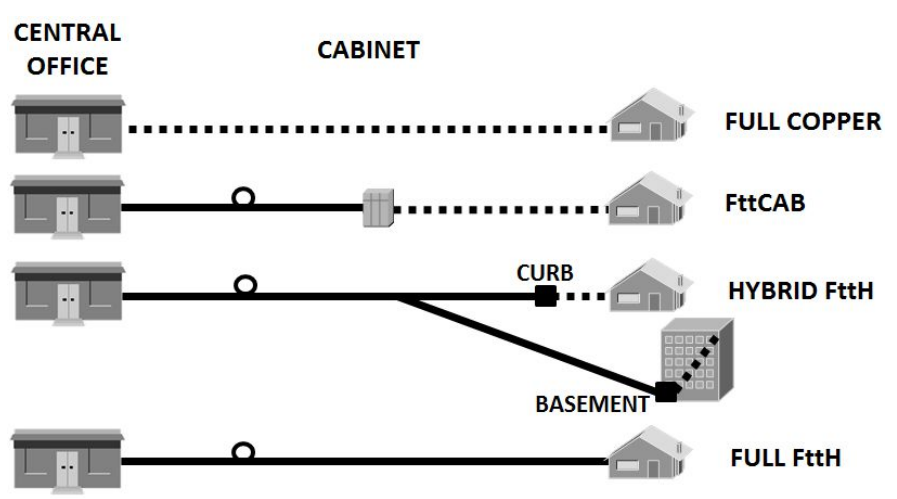

Fig. 1. Four topologies

Here a new node is realized within 200 meter of each house connected. This 200 meter is the assumed maximum distance that G.Fast brings value. We assume that a branching point in the existing copper connections is chosen to place the new active equipment. Technical issues like modulation and power supply are considered in other work of the CELTIC/4GBB project [1]. The new nodes have to be connected by a fibre connection. We argue in this paper that there have to be made three main choices before designing the network. If these three choices all have two options, we end up with 8 possible rollout scenarios that we all elaborate in this paper.

In the remainder of this paper we first describe our starting position of the copper network and the main choices that have to be made by the designer of the network. Next we will elaborate the various combinations of those choices and explore literature for the mathematical approach for those combinations. At the end we shall discuss a real case from two cities in the Netherlands, Amsterdam and The Hague.

\section{IDENTIFYING THE OPTIONS}

In this section we will present the framework based on three questions and elaborate the eight planning options that result from these questions. Next we will discuss shortly the choice between a tree and a ring based network structure. 


\section{A. Three Questions}

When we look in more detail to this next part of the copper network we see a situation as shown in Fig. 2. This is a typical situation in the last mile of the Dutch copper network: a heavily branched network, with at the right side a cabinet. In this network new network nodes have to be placed for the G.Fast technology. To do this, possible locations for these network nodes have to be determined, logical places are the dots in the figure, the branching points of the network. We assume that it is known which houses are connected to these locations at which distance. Now one should decide which locations will be used and how they are connected to a fibre node in the most economical way.

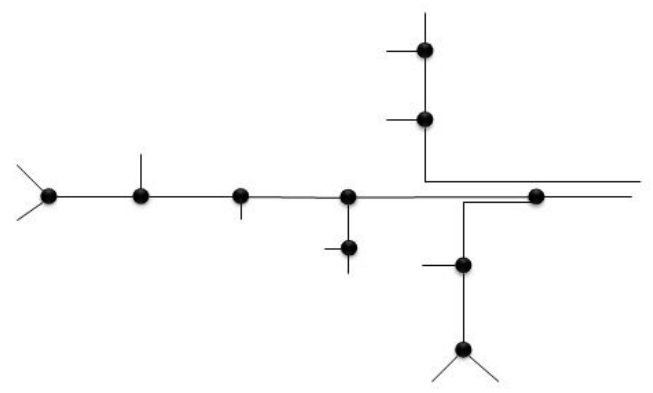

Fig. 2. Typical last mile in the Netherlands

The three questions that form the basis of our framework are:

1) Should all houses be reached from a Hybrid Fibre node within a fixed distance, or a fixed percentage of houses, or do we have a fine for every house not connected within that certain distance? We distinguish:

a) All houses must be connected, a fine is considered otherwise.

b) A certain percentage has to be within the defined distance.

2) Does the node have a capacity restriction?

a) Yes

b) No

3) How are the nodes connected:

a) Tree or star structure

b) Ring structure

\section{B. Elaborate the Planning Options}

In the previous section we had 3 choices to be made, each having 2 possible answers. This leads to $\left(2^{3}=\right)$ eight possible roll-out scenarios that are in theory all thinkable. In this section we will discuss all scenarios and propose a mathematical approach to each planning problem. We refer to each scenario with a three letter acronym, each representing the chosen answers to the questions. As example, the scenario AAA refers to the case where all questions were answered by option (a): all houses connected, the node has a capacity restriction and the nodes are connected by a tree or star structure. The eight possible roll out scenarios are then:
- AAA: CFLP plus MSP: The scenario AAA refers to the case where all houses have to be connected, the node has a capacity restriction and the nodes are connected by a tree structure. This problem can be seen as the case where from several possible facilities with a certain maximum capacity we have to choose a subset of those facilities and assign customers to a facility such that all customers are served by one facility at minimal cost. This is a Capacitated Facility Location Problem (CFLP). Next the opened facilities have to be connected with the central point (cabinet, central office) in a star structure. To do this the shortest path between the central point and the opened facilities can be determined, but to reduce the cost of digging it is more economical to take the minimal spanning tree between all the facilities and the central point by solving a Minimal Spanning tree Problem (MSP).

- AAB: CFLP plus VRP: The scenario AAB refers to the case where all houses are connected, the node has a capacity restriction and the nodes are connected by a (multiple) ring structure. This looks like the previous problem, only now the routing comes into scope. The central point uses ring structures to serve the opened nodes in a shortest cycle. Which ring has to serve which node and what is the shortest path the ring has to go? This is a Vehicle Routing Problem (VRP), or if there is a maximum number of nodes that can be connected in one ring a Capacitated Vehicle Routing Problem (CVRP).

- ABA: standard FLP plus MSP: The scenario ABA refers to the case where all houses are connected, the node does not have a capacity restriction and the nodes are connected by a tree structure. This is a standard or uncapacitated Facility Location Problem (FLP). Again the Minimum Spanning Tree Problem can be used to connect te opened facilities.

- ABB: standard FLP plus VRP: The scenario ABB refers to the case where all houses are connected, the node does not have a capacity restriction and the nodes are connected by a ring structure. This is a uncapacitated Facility Location Problem in combination with a Vehicle Routing Problem.

- BAA: activation problem plus MSP: The scenario BAA refers to the case where a certain percentage of the houses have to be within the defined distance, the node has a capacity restriction and the nodes are connected by a tree structure. This is the same problem as discussed in [2] for VDSL cabinet activation, combined with the Minimum Spanning Tree Problem to connect the opened facilities.

- BAB: activation problem plus VRP: The scenario BAB refers to the case where a certain percentage of the houses have to be within the defined distance, the node has a capacity restriction and the nodes are connected by a ring structure. This is again the activation problem, combined with the Capacitated Vehicle Routing Problem (CVRP) to connect the opened facilities.

- BBA: activation problem plus MSP: The scenario BBA refers to the case where a certain percentage of the houses have to be within the defined distance, the node does not 
have a capacity restriction and the nodes are connected by a tree structure. This is the activation problem, now with infinite capacity on the nodes. Again combined with the Minimum Spanning Tree Problem to connect the opened facilities.

- BBB: activation problem plus VRP: The scenario BBA refers to the case where a certain percentage of the houses have to be within the defined distance, the node does not have a capacity restriction and the nodes are connected by a ring structure. This is the activation problem, now with infinite capacity on the nodes combined with the Capacitated Vehicle Routing Problem (CVRP) to connect the opened facilities.

If we look at these eight roll-out scenarios and the identified standard problems we can summarize this in the following six mathematical main problems:

1) scenario AAX: Capacitated Facility Location Problem (CFLP).

2) scenario ABX: Uncapacitated Facility Location Problem (FLP).

3) scenario BAX: Activation Problem (CACT).

4) scenario BBX: Activation Problem with infinite node capacity (ACT).

5) scenario XXA: Minimum Spanning Tree Problem (MSP).

6) scenario XXB: (Capacitated) Vehicle Routing Problem (CVRP).

The total framework can now be summarized in a flow diagram, as depicted in Fig. 3.

\section{Ring or Star}

One of the choices to be made was the choice between a star or tree and ring topology. In the Netherlands ring structures are common, but in other European countries star or tree topologies are conventional. Mostly cost are the driver for this choice. Ring topology deliver a much higher reliability however, and the break-even costs (in terms of distance of digging) where both topologies are equally expensive is reached fairly rapidly (ring vs star) or are close all the time (ring vs tree).

Theoretically we could derive the break-even point between the star and the ring structure very simply. Say we have $n$ nodes, all of them at the distance $r$ of a centre point. Connecting them with a star structure will cost $n * r$. If we use a ring structure, all nodes are on the ring with radius $r$, the total costs are

$$
\frac{n-1}{n} * 2 * \pi * r+2 * \pi .
$$

The break even point is where

$$
n * r=\frac{n-1}{n} * 2 * \pi * r+2 * \pi .
$$

This is true when

$$
n=\pi+1+\sqrt{\pi^{2}+1}=7.44 .
$$

This means that if we want to connect 8 or more nodes, a ring structure is cheaper.

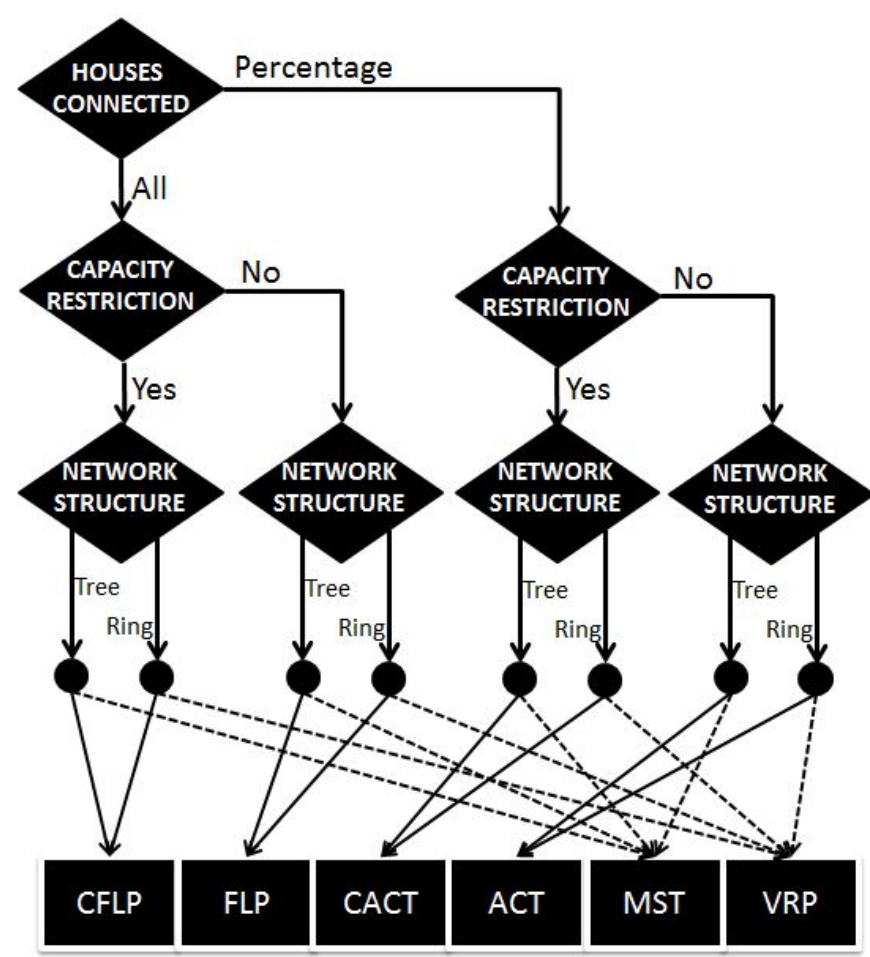

Fig. 3. Framework flow diagram

However, it is obvious that the nodes will not be distributed such that they are all at distance $r$ from the central office. To find the relation between the number of nodes and the digging length of the three network structure options we simulated 1000 situations where $n$ nodes are placed randomly within an area with dimension $100 \times 100$. The central node is placed at $(x, y)=(50,50)$. For each situation we connected the $n$ points with the central node in a star structure, in a tree structure, in a ring structure and in a ring structure with at most 10 nodes per ring. The ring is created solving a TSP using a generic insertion algorithm and 2-opt algorithm. The ring with capacity constraint is created solving a CVRP using Clark and Wright savings algorithm [3]. The tree is calculated using Prim's algorithm [4]. The results are shown in Fig. 4. Here we see that the break even point for star vs ring is between 6 and 7 nodes and the that the tree is always slightly cheaper than the ring structures. The owner of the network has to weight this against the differences in reliability of the structures.

\section{ElABORATE THE STANDARD PROBLEMS}

In the previous section we identified six mathematical main problems that appear when effect the eight roll-out scenarios. In this section we will give for each problem an overview of literature dealing with that problem. 


\section{Length of total connection}

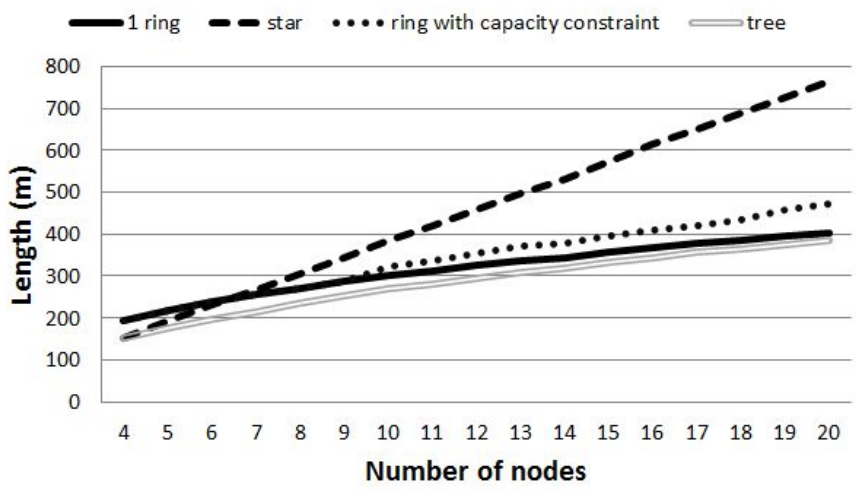

Fig. 4. Digging distances

\section{A. Uncapacitated Facility Location Problem}

The FLP revolves around the following problem: given a set $F$ of facilities, a set $D$ of customers, costs $f_{j}$ for opening facility $j \in F$ and connection costs $c_{i j}$ for connecting the customer $i$ with facility $j$ : which subset of facilities in $F$ needs to opened and which customers have to be connected with which open facility, in order to minimize the costs. A facility is considered open when at least 1 customer is serviced by this facility. Opening a facility and servicing customers involves costs. To coop with the maximum distance of 200 meters one should express that in the cost parameter $c_{i j}$.

Literature (e.g. [5]) makes a distinction between several different types of FLP. The difference is important, as the known heuristics used cannot be the same for all types. The first important type here is the Uncapacitated Facility Location Problem (UFLP). The assumption there is that the capacity of a facility, or the number of customers which can be serviced, is infinite, and the costs of opening a facility are set. So the opening costs of a facility are not determined by the number of customers serviced.

Again in [5] a metric Uncapacitated Facility Location Problem (UFLP) is discussed. The connection cost is metric as they are symmetrical and meet the triangle inequality. The article discusses first the JMS heuristics. The JMS heuristics work as follows:

1) At the start, all customers are unconnected and all facilities closed, and the budget of every customer $i$, noted with $B_{i}$ is equal to 0 . In every step every customer $i$, based on his actual budget, makes an offer to each closed facility $j$. The size of the offer equals to $\max \left(B_{i}-c_{i j}\right)$ if customer $i$ is not connected and is equal to $\max \left(c_{i j^{\prime}}-c_{i j}, 0\right)$ if customer $i$ is connected to another facility $j^{\prime}$.

2) If there is an unconnected customer, increase the budget of each unconnected customer by the same value, until one of the following events occurs:

a) If for an unopened facility $j$, the total offer which facility $j$ receives from all customers is equal to the

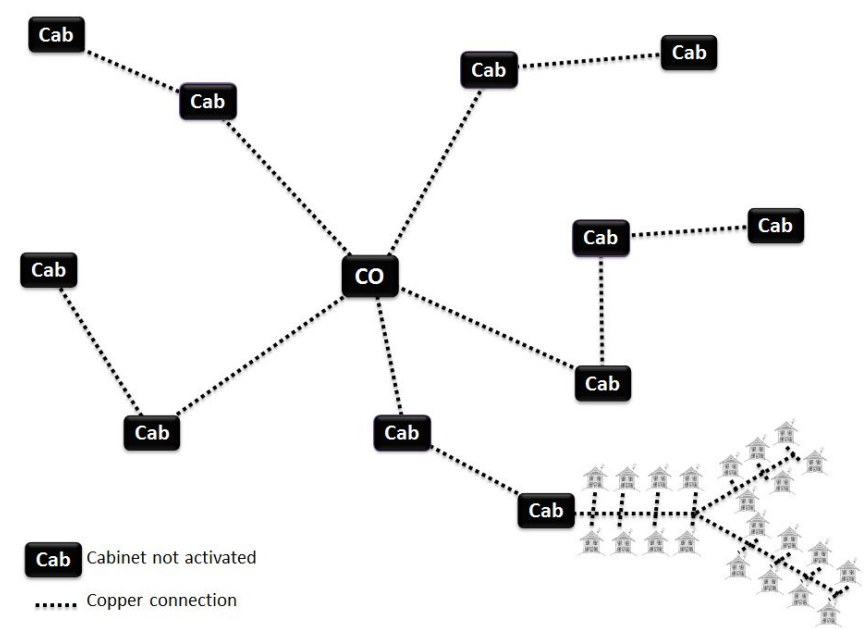

Fig. 5. Starting point

costs of opening facility, then we open facility $j$ and for each customer $i$ (serviced or not serviced) that has an offer to facility $j$ greater than 0 , we connect customer $i$ with facility $j$.

b) If for a non-serviced customer $i$ and an already opened facility $j$ the budget of customer $i$ equals the connection costs $c_{i j}$, then we connect customer $i$ with facility $j$.

Next they present a more complex, but also more efficient algorithm.

\section{B. Capacitated Facility Location Problem}

Where the UFLP can be solved relatively easy by a good and simple heuristics, adding capacity constraints to the facilities makes the problem much more difficult. Most research on the CFLP has focused on the development of efficient solution algorithms, based on branch-and-bound techniques, Lagrangian relaxation, Benders decomposition etcetera, see for example [6], [7] and [8]. Ref. [9] defines the Capacitated Connected Facility Location Problem (CapConFL) for a similar problem. A nice local search heuristic can be found in [10]. An other possibility is to use the solution to the Activation Problem of the next section, with $100 \%$ customers connected.

\section{Activation Problem}

In [2] this problem is discussed for the FttCab roll-out. This works for both the activation problem with and without infinite node capacity. The problem there is: which cabinets must be activated in order to reach the desired percentage of households at minimal costs? Fig. 5 shows the starting point. All cabinets $(\mathrm{Cab})$ are connected through copper with the Central Office (CO). Several residences are connected to the cabinet; this is only shown for one cabinet in the illustration. Now a subset of the cabinets needs to be activated in order to reach the intended number of households over copper from an activated cabinet within the set distance, see Fig. 6. In fact, this is a generalization of the CapConFL.

The proposed heuristics is shown in Fig. 7. It starts with a logical, allowed, solution, in which all cabinets are activated 


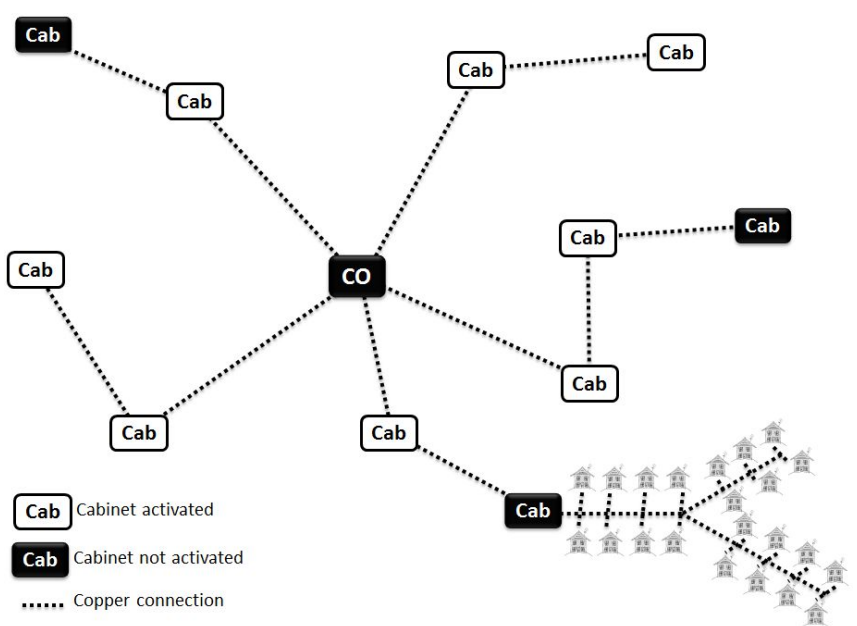

Fig. 6. Which cabinets are activated?

in step 1. Next in step 2, all possible cascade arrangements are determined and the savings of this arrangement (call it $B)$ as well as the number of customers which as a result are positioned outside the desired distance of, here, 200 meter (call it $K$ ) are reviewed. Next, the solutions which generate a saving $(B>0)$ can be sorted by two possible characteristic: $B$ and $B / K$. In step 3 we realize the solutions with the largest (negative) value of $B$ or $B / K$, until the requirement of e.g. $90 \%$ of the customers is reached. In step 4 we perform a 2 opt approach to improve the solution. The 2-opt methodology was originally presented for solving the travelling salesman problem (TSP), see [11]. If step 4 results in a swap, we try to find a new improvement; if no swap could be found the best solution was found. We showed that this algorithm is very fast.

\section{Minimum Spanning Tree Problem}

Given a connected, undirected graph, a spanning tree of that graph is a connected sub graph, connecting all the vertices of the original graph. If the edges have a weight assigned, we can use these weight to compute the weight of the spanning tree, the sum of the weights of the edges in that spanning tree. A minimum (weight) spanning tree is then a spanning tree with weight less than or equal to the weight of every other spanning tree. A solution to MSP can be found in [4]. An alternative is the method of Kruskal. A nice comparison can be found in [12]. Prim's algorithm is quite simple:

1) Take some arbitrary start node $s$. Initialize tree $T=s$.

2) Add the cheapest edge, which has one vertex in $T$ and one vertex not in $\mathrm{T}$, to $\mathrm{T}$.

3) If $T$ spans all the nodes the Minimum Spanning Tree is ready, else repeat step 2 .

\section{E. (Capacitated) Vehicle Routing Problem (CVRP)}

The Vehicle Routing problem comes from logistics and describes the problem that clients have to be serviced from (one or more) depots, using one or more vehicles that might have a certain capacity constraint. The question in this problem is which client is serviced by which vehicle from which depot

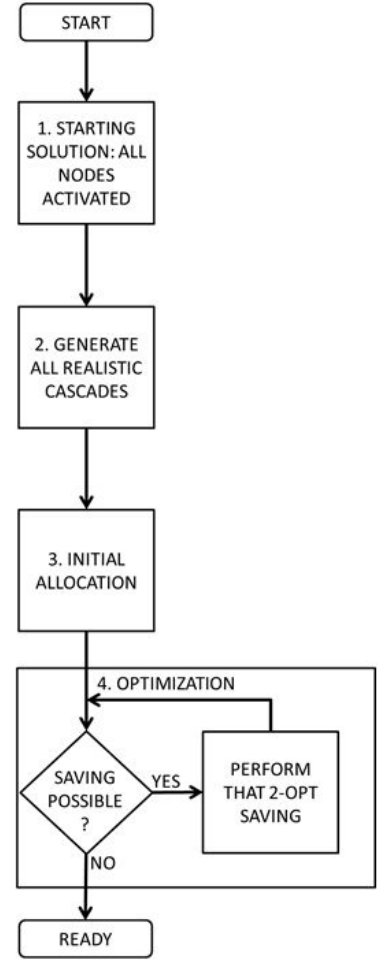

Fig. 7. Overview of the heuristics

and what is the shortest route the vehicle will drive. Two main questions in our problem will be: which node is serviced by which ring and how does the ring run physically. To solve these two problems together the best-known approach is the "savings" algorithm of Clarke and Wright. Its basic idea is very simple, as described in [3]: 'Consider a depot $D$ and $n$ demand points. Suppose that initially the solution to the VRP consists of using $n$ vehicles and dispatching one vehicle to each one of the $n$ demand points. The total tour length of this solution is, obviously, $2 \sum_{i=1}^{n} d(D, i)$. If now we use a single vehicle to serve two points, say $i$ and $j$, on a single trip, the total distance travelled is reduced by the amount

$$
\begin{aligned}
s(i, j)= & 2 d(D, i)+2 d(D, j) \\
& -[d(D, i)+d(i, j)+d(D, j)] \\
= & d(D, i)+d(D, j)-d(i, j)
\end{aligned}
$$

The quantity $s(i, j)$ is known as the "savings" resulting from combining points $i$ and $j$ into a single tour. The larger $s(i, j)$ is, the more desirable it becomes to combine $i$ and $j$ in a single tour. However, $i$ and $j$ cannot be combined if in doing so the resulting tour violates one or more of the constraints of the VRP.' Where $d(i, j)$ is the distance function.

However, to fully exploit the reliability gain of a ring structure, all the elements (paths) of the ring should be independent. The ring should not use the same trench or cable twice (or more). This is not taken into account in a regular CVRP solutions, like Clarke and Wright.

Kalsch et al. [13] developed a mathematical model and a heuristic approach for embedding a ring structure in a fibre network, that takes into account the following restrictions: 
ensuring a ring structure, a maximum number of nodes in a ring, each node in exactly one ring, and that the ring uses each edge only once. It is, however, hard to draw conclusions on the performance of their approach, since no further information is given on the data used for a test case. Another important disadvantage of their method is that no real attention is paid to the clustering of the nodes to the rings. They indicate clustering is part of the problem, but do not really treat it in there article and they go directly to the routing part of the problem.

For a similar problem in the designing of a FttCab network the two problems, clustering and routing, were solved by us in succession. First the nodes were clustered in groups and then a ring is created through these groups (see [14]). In our current research we are developing a method to solve both steps together, within reasonable calculation times.

\section{CASES}

We performed scenario BBA to two cities in the Netherlands, Amsterdam and The Hague, using the activation algorithm [2] and Prim's algorithm [4]. We assume that the cabinets already have a fibre connection, so our focus is the part of the network between the cabinet and the home connection. The Amsterdam case has 150,058 branching points in that area, The Hague has 89,076 branching points. Those branching points are the potential spots to place the new equipment. In Fig. 8 an example is shown; a part of Amsterdam with all the splices and cabinets. The pictures comes from the GIANT/PLANXS tool of TNO, which performs FttCab and FttCurp planning problems. In both cities we want to connect at least $99 \%$ of the customers within 200 meter to a G.Fast node. Each G.Fast node is placed in a manhole. We can place each combination of 16-port and 48-port G.Fast equipment (G.Fast multiplexer) in the manhole.

The problem that is solved by the activation algorithm is the following. The central question is: which nodes should be activated in order to reach the desired percentage of households at minimal costs? A household is reached when the distance over copper is less than a chosen length, here 200 meter. Households which meet this requirement are said to meet the distance requirement. Here we describe the problem as a mathematical model. The chosen structure is that of an Integer Programming Problem. We first define the decision variables:

$$
\begin{gathered}
x_{i j}= \begin{cases}1 & \text { if node } i \text { is handled by node } j \\
0 & \text { otherwise. }\end{cases} \\
y_{j}= \begin{cases}1 & \text { if node } j \text { is activated, } \\
0 & \text { otherwise }\end{cases}
\end{gathered}
$$

The input we need is described by the following parameters:

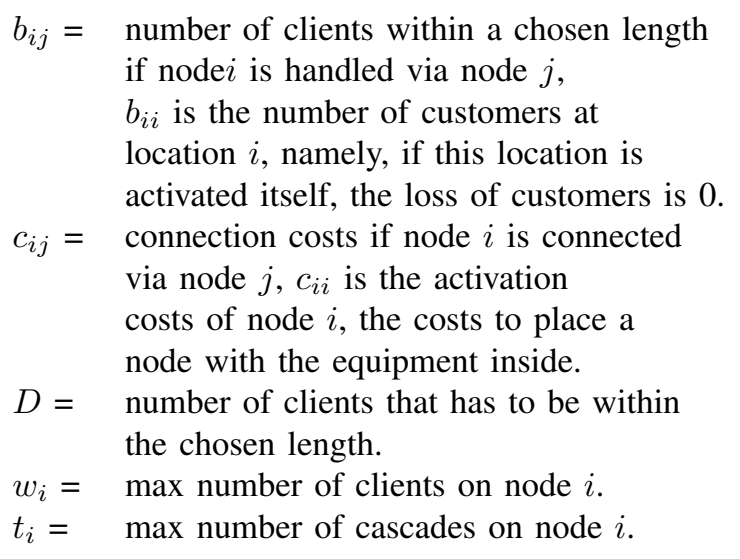

The indices $i$ en $j$ are defined as the nodes $1, \ldots, n$. In (1) it is stated what we want to reach: the decision variables need to be chosen in such a manner that the objective function, total costs for activation and connection, are minimal.

$$
\min _{x, y} \sum_{i=1}^{n} \sum_{j=1}^{n} c_{i j} x_{i j}+\sum_{j=1}^{n} c_{j j} y_{j}
$$

The constraints of this problem are:

$$
\begin{gathered}
\sum_{j=1}^{n} x_{i j}=1 \quad i=1, \ldots, n \\
\sum_{i=1}^{n} \sum_{j=1}^{n} b_{i j} x_{i j} \geq D \\
y_{j} \geq \frac{\sum_{i=1}^{n} x_{i j}}{n} \quad j=1, \ldots, n \\
\sum_{i=1}^{n} x_{i j} \leq t_{j} \quad j=1, \ldots, n \\
\sum_{i=1}^{n} b_{i i} x_{i j} \leq w_{j} \quad j=1, \ldots, n \\
x_{i j}, y_{j} \in\{0,1\} .
\end{gathered}
$$

The conditions that need to be met are:

(2) This condition says that each node has to be dealt with via exactly one (other) node.

(3) The total number of customers connected within the chosen length, 200 meter, to the node needs to be larger than or equal to $\mathrm{D}$.

(4) If a node is handled via node $j$, node $j$ needs to be activated.

(5) No more than $t_{j}$ nodes may be cascaded to one other node .

(6) No more than $w_{j}$ customers may be within a cascade.

(7) Both $x_{i, j}$ and $y_{j}$ are binary variables.

There are two important constraints:

1) One arriving cable at the G.Fast cannot be spread over 2 G.Fast multiplexers.

2) Maximum distance over copper to the active point

For the first constraint look at the example in Fig. 9, two cables arrive at node $\mathrm{A}$, one with 14 connections and one 


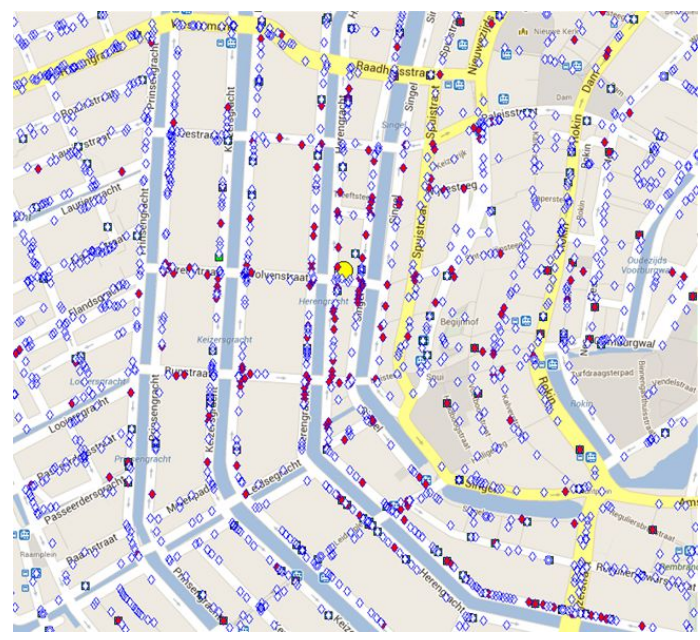

Fig. 8. Part of Amsterdam showing all the splices (open dots) and cabinets (closed dots)

TABLE I

COSTS INPUT

\begin{tabular}{|l|r|}
\hline G.Fast multiplexer & $€ 25$ per port \\
\hline G.Fast Manhole & $€ 500$ \\
\hline Digging and cables & $€ 25$ per meter \\
\hline
\end{tabular}

cable with 9 connections. If the capacity of the multiplexer is 16 , node $\mathrm{A}$ can be used to handle both cables with two multiplexers. However, these $(14+9=) 23$ cables arrive at node $B$ in one cable. This cable cannot be handled with one multiplexer, thus these cables should be handled by an activated node before node $B$. In the activation problem this situation should be depicted in the parameter $b_{i j}$, meaning: handling the connections of node $i$ by node $j$ keeps $b_{i j}$ connections within the desired distance. However the distance from the home connections to $B$ or $C$ might be less than the chosen maximum copper length 200 meter, we have to make $b_{i j}$ with $\mathrm{i}=\mathrm{A}$ or lower in the network and $j=B$ or higher in the network equals zero to prevent handling the connections at $B$ or $C$. If we have also a 48 port multiplexer, this connection can be handled by node $B$ or $C$.

Also the length constraint, the second constraint, should be depicted in the parameter $b_{i j}$. Here, with a node capacity of 48 connections and equipment capacity of 48 connection, $b_{A A}=$ $23, b_{A B}=23, b_{A C}=23$ but $b_{A C a b}=0$. The length to the cabinet is more than 200 meters. The minimal cost selection of new locations for these G.Fast nodes is then connected by a Minimum Spanning Tree. We assume costs as shown in Table I.

The calculation time for the case Amsterdam is 50 seconds, consisting of:

- 14 Spanning Tree calculations: 1 seconds

- Database interaction and data handling: 25 seconds

- Solving 2745 Activation Problems: 24 seconds

The activation problem activates 7,366 new G.Fast nodes, out of the possible 150,058 , for 490,000 connections in

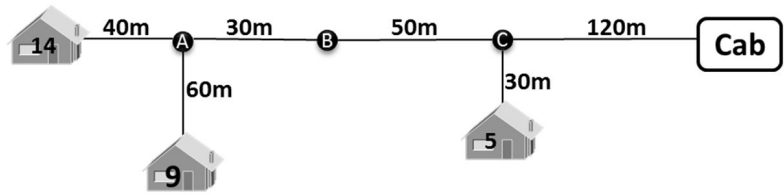

Fig. 9. Example of cable with connections

TABLE II

RESULTS AMSTERDAM

\begin{tabular}{|l|r|r|}
\hline Digging (meter) & 686,106 meter & $€ 17,152,650$ \\
\hline Equipment (ports) & 661,024 ports & $€ 16,525,600$ \\
\hline Manholes (new node) & 7,366 & $€ 3,683,000$ \\
\hline Total costs (euro) & & $€ 37,361,250$ \\
\hline Per connection (euro) & & $€ 76.19$ \\
\hline
\end{tabular}

Amsterdam ${ }^{1}$. The results of this calculations are in Table II. This means we have a port utilization ${ }^{2}$ of $74 \%$ and an average digging distance per node of 93 meters. For various distances, the costs per home connected is depicted in Fig. 10. Note that we do not make extra nodes, next to the existing branching points, thus the minimum distance is restricted by the length of the last piece of copper in the path towards the houses. A copper length of 25 meter does not indicate that all copper lengths are lower, but only those connections who can physical realize this. Otherwise the graph is expected to increase faster when decreasing the distance. The trend line indicating this in the figure is an estimation of the real relation, based on a logarithmic trend.

For The Hague the results are in Table III and in Fig. 11. Here we have 288000 connections, resulting in a port utilization of $73 \%$ and an average digging distance per node of 122 meters. The difference between Amsterdam and The Hague are explained by the existing copper infrastructure. Amsterdamregion has already $60 \%$ of the homes within 200 meters of the cabinet, and Amsterdam-Centre even 75\%. The Hague only has $28 \%$ of the connections within 200 meters.

\footnotetext{
${ }^{1}$ There are more ports than connections, due to the fixed number of ports per multiplexer.

${ }^{2}$ Number of connections divided by number of ports.
}

TABLE III

Results The Hague

\begin{tabular}{|l|r|r|}
\hline Digging (meter) & $1,058,350$ meter & $€ 26,458,750$ \\
\hline Equipment (ports) & 395,328 ports & $€ 9,883,200$ \\
\hline Manholes (new node) & 8,656 new nodes & $€ 4,328,000$ \\
\hline Total costs (euro) & & $€ 40,669,950$ \\
\hline Per connection (euro) & & $€ 141.15$ \\
\hline
\end{tabular}




\section{REFERENCES}

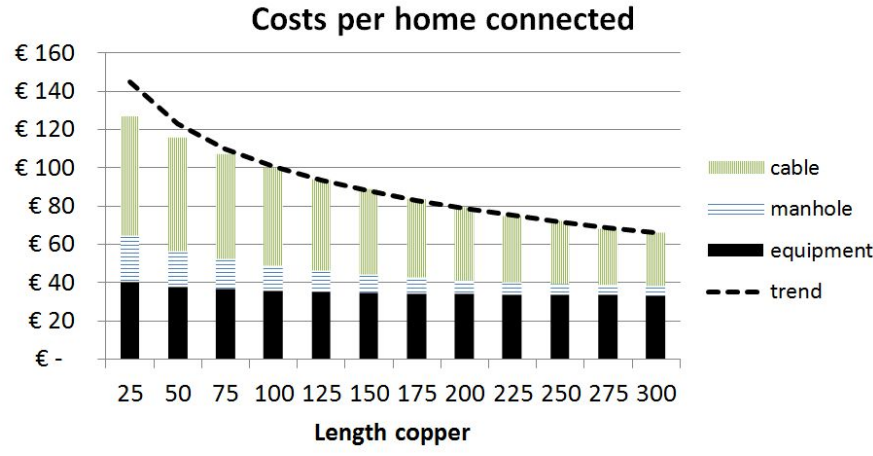

Fig. 10. Costs of connection in Amsterdam

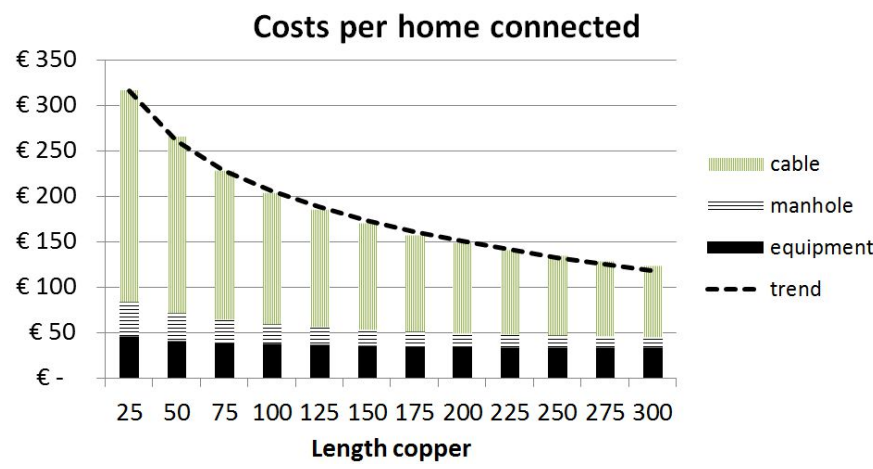

Fig. 11. Costs of connection in The Hague

\section{SUMmARY AND CONCLUSIONS}

In this paper we looked at the planning of the Hybrid FttH variant using G.Fast as technology, where the fibre is brought to a place in the street, also known as Fibre to the Curb. To realize FttCurb using G.Fast a next step in bringing fibre to the houses is needed. Here a new node is realized within 200 meter of each house connected. We assumed that a branching point in the existing copper connections is chosen to place the new active equipment. The new nodes have to be connected by a fibre connection. We presented a framework that is based on three main choices before designing the network. If these three choices all have two options, we end up with eight possible planning options and 6 main mathematical challenges, which we all elaborated in this paper, showing the mathematical approach for all of these options. For one of the options we showed the results of a real life case, the planning of FttCurb in Amsterdam and The Hague.

\section{ACKNOWLEDGMENT}

The author wishes to thank Rob van der Brink and Harrie van der Vlag for providing the data for the cases.
[1] CELTIC-4GBB. [Online]. Available: www.4gbb.eu

[2] F. Phillipson, "Fast roll-out of fibre-to-the-cabinet: optimal activation of cabinets," In preparation, 2013.

[3] R. C. Larson and A. R. Odoni, Urban Operations Research. Prentice Hall, 1981.

[4] R. C. Prim, "Shortest connection networks and some generalizations," Bell Systems Technical Journal, vol. 36, p. 13891401, 1957.

[5] M. Mahdian, Y. Ye, and J. Zhang, "Approximation algorithms for metric facility location problems," SIAM J. Comput., vol. 36, no. 2, pp. 411432, 2003.

[6] K. Holmberg, M. Rnnqvist, and D. Yuan, "An exact algorithm for the capacitated facility location problems with single sourcing," European Journal of Operational Research, 1999.

[7] S. Melkote and M. S. Daskin, "Capacitated facility location/network design problems," European Journal of Operational Research, vol. 129, pp. 481-495, 2001.

[8] K. Aardal, D. Shmoys, and E. Tardos, "Approximation algorithms for facility location problems," in Proceedings of the 29th ACM Symposium on Theory of Computing, 1997.

[9] S. Gollowitzer, B. Gendron, and I. Ljubić, Capacitated Network Design with Facility Location. CIRRELT, 2012.

[10] V. Arya, N. Garg, R. Khandekar, A. Meyerson, K. Munagala, and V. Pandit, "Local search heuristics for k-median and facility location problems," in Proceedings of the 33rd ACM Symposium on Theory of Computing, 2001.

[11] G. Croes, "A method for solving traveling salesman problems," Operations Research, vol. 6, pp. 791-812, 1958.

[12] H. J. Greenberg, "Greedy algorithms for minimum spanning tree," University of Colorado at Denver - http://glossary.computing. society.informs.org/notes/spanningtree.pdf, Tech. Rep., 1998.

[13] M. T. Kalsch, M. F. Koerkel, and R. Nitsch, "Embedding ring structure in large fiber networks," in XVth International Telecommunications Network Strategy and Planning Symposium (NETWORKS), 2012.

[14] F. Phillipson, "Efficient clustering of cabinets at FttCab," in The 13th International Conference on Next Generation Wired/Wireless Advanced Networking (NEW2AN 2013), St.Petersburg, Russia, 2013.

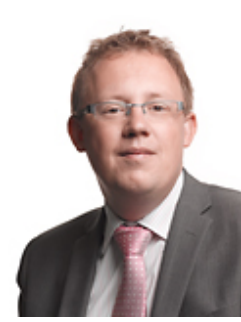

Frank Phillipson (1973), studied Econometrics at the Vrije Universiteit Amsterdam, and wrote his Master's thesis in the field of Operations Research in 1996. In the same year he joined the Delft University of Technology to follow the Post-Doctoral course Mathematical Design Engineering with a strong focus on application of Operations Research techniques in networks. From 1998 until 2003 he was employed at KPN Research. In 2002, KPN placed its research department in TNO, the largest applied research institute in the Netherlands, where Frank is currently working in the department 'Performance of Networks and Systems'. There he is particularly working in the field of planning of ICT/telecom and electricity networks. In addition to this main topic, he has worked on projects for financial and economic models relating to telecom business. This has provided him a good overview of the technical as well the economic impact of network planning and dimensioning. Frank Phillipson is co-author of several papers and has supervised many Masters students working on their thesis at TNO. 